\title{
Preliminary studies into the effect of environmental degradation on the characterisation of automotive clear coats by attenuated total reflectance infrared spectroscopy
}

\author{
Georgina Sauzier, ${ }^{a}$ Mark Maric, ${ }^{a}$ Wilhelm van Bronswijk, ${ }^{a}$ and Simon W. Lewis ${ }^{a *}$ \\ s Received (in $X X X, X X X)$ Xth $X X X X X X X X X 20 X X$, Accepted $X$ th $X X X X X X X X X 20 X X$ \\ DOI: $10.1039 / b 000000 x$
}

This paper presents a study into the impact of environmental weathering on the chemometric classification of automotive clear coats. These studies were conducted using attenuated total reflectance infrared spectroscopy, in conjunction with a chemometric model capable of predicting the country of vehicle manufacture for a sample based upon its infrared spectrum. Testing of three samples exposed to 10 environmental conditions found the model to be robust to weathering over at least an eight-month period. However, analysis of 12 samples of unknown prior history found that extreme degradation over at least a 10-year period, or the presence of a post-manufacture (respray) coating, could result in misclassification when relying solely on analysis of the clear coat. It is therefore important that crosssections comprising the full layer sequence of the paint system are obtained wherever possible, as analysis of the underlying layers may give more comprehensive characterisation.

\section{${ }_{15}$ Introduction}

In recent years there has been an increased emphasis on establishing more scientifically rigorous approaches to the interpretation of forensic evidence, particularly in regards to trace evidence such as glass, fibres or paint. ${ }^{1}$ Automotive paint is a

20 complex multi-layered system that is commonly encountered as trace evidence at the scenes of automobile-related incidents. ${ }^{2,3}$ In cases where automotive paint is the only form of physical evidence encountered at the scene, its analysis may facilitate the procuring of investigative leads. ${ }^{4}$

25 Analysis of automotive paint is typically conducted as a sequence of methods characterising various physical or chemical properties of the sample. ${ }^{4-6}$ This information may be used to identify the source of the vehicle, either through comparison to a reference sample of known origin, or through library searching of 30 a database. Although various instrumental methods may be utilised, infrared spectroscopy is widely regarded as the most powerful non-destructive technique for the analysis of paint traces. ${ }^{7-9}$ In particular, attenuated total reflectance (ATR) infrared spectroscopy has frequently been employed for the forensic 35 analysis of automotive paint samples, and has been demonstrated to give discrimination between clear coat layers of different vehicles. ${ }^{10-12}$ However, when considering the results of spectroscopic analysis, forensic examiners often still rely on visual comparisons of complex spectra, and thus lack a statistical 40 basis upon which to evaluate evidential significance. ${ }^{1,13,14}$

Chemometrics allows objective conclusions to be drawn from experimental data through the use of statistical methods. ${ }^{15}$ In addition to identifying patterns or correlations within complex datasets, chemometric methods can improve discrimination 45 between samples whilst also addressing concerns regarding human bias in forensic examinations. ${ }^{1,16,17}$ While the application of spectroscopic techniques to the analysis of automotive paint samples has been well documented, chemometrics has not been routinely employed to interpret the resultant data. ${ }^{9}$

Previous research by Maric et al. investigated the chemometric characterisation of automotive clear coats analysed by ATR FTIR spectroscopy. ${ }^{18}$ The clear coat is the uppermost layer applied to the automotive paint system, and contains ultraviolet (UV) absorbers and light stabilisers designed to protect the exterior ${ }_{55}$ surface of the vehicle from UV degradation and weathering. ${ }^{19,} 20$. Principal component analysis (PCA) and linear discriminant analysis (LDA) were employed to analyse over 700 infrared spectra from the clear coat layers of 130 vehicles, covering a range of both Australian and international manufacturers. This ${ }_{60}$ resulted in the development of a chemometric model capable of classifying unknown clear coat samples into one of nine identified classes. These classes were subsequently linked to the country of vehicle origin, and in some instances to the manufacturer or manufacturing plant of the vehicle. This model ${ }_{65}$ could potentially be utilised to generate investigative leads from questioned samples by narrowing down the field of search for a suspect vehicle, or by excluding vehicles from further inquiry. ${ }^{18}$

While the approach described by Maric et al. has great potential for forensic investigations, the samples used were taken 70 from factory-finish or near-factory-finish vehicles, which had not undergone any significant weathering or environmental exposure. Hence, while the model has been shown to give accurate information regarding relatively new samples, its applicability to 'real' samples that may have undergone extensive weathering has 75 yet to be evaluated.

The potential for environmental factors such as temperature, UV irradiation or atmospheric pollutants to alter the chemical composition of automotive paint on vehicles over time has been 
well documented in open literature, ${ }^{21-23}$ including a ${ }_{80}$ comprehensive review by Mohseni et al. ${ }^{24}$ However, these studies have been predominantly conducted from a manufacturer viewpoint, with the intent of maximising the service life of automotive coatings.

There is a paucity of research in the open scientific literature 85 considering the impact of environmental degradation on the characterisation of paint samples within a forensic context. Chang et al. investigated the effect of weathering on IR spectral database searches of automotive basecoats and clear coats for forensic purposes, over a three-year period. ${ }^{25}$ They found that while the

90 majority of coatings could still be correctly matched to nonweathered exemplars throughout the three-year period, some samples containing acrylic- and alkyd-melamine binders exhibited changes in their spectra as a result of environmental exposure. It should be noted however, that the samples utilised in 95 this study were mounted in an embedding resin prior to analysis. Subsequent research revealed the potential infiltration of embedding media into paint samples, causing interfering bands in the resultant IR spectra which may have impacted upon the results of the previous study. ${ }^{26}$

100 This paper presents a preliminary study into the effects of environmental exposure upon the characterisation of automotive clear coats analysed by ATR FT-IR spectroscopy, in conjunction with multivariate statistics as previously utilised by Maric et al. This investigation included samples deliberately treated to

105 undergo degradation, in addition to samples where the prior history was unknown, as may be the case for a questioned sample submitted for forensic analysis.

\section{Materials and Methods}

\section{Sample Collection and Preparation}

110 Samples for degradation studies were obtained through a car sunroof fitting company (Prestige Sunroofs W.A., Perth, Australia) from roof panels removed during sunroof installation. The Vehicle Identification Number (VIN) of each vehicle was recorded. Three samples were attached to steel struts on a

115 building roof at Curtin University to undergo environmental exposure. Infrared spectra of each sample were also obtained prior to exposure in order to track subsequent changes. The samples were exposed over an eight-month period, removing paint chips for infrared analysis every two months. A scalpel was

120 used to pry segments of the paint off the underlying metal, ensuring that all layers were present.

12 external test samples with unknown prior history of environmental exposure were obtained from eight vehicles, through the Western Australia Police (WAPOL) and an 125 automotive repair company (Maddington Auto Wreckers, Perth, Australia). As the VIN was not available for all vehicles, the make and model for each was recorded. These samples originated from vehicles manufactured pre-2000 with visible signs of weathering, and were treated as a rigorous test to establish the

130 limitations of the existing model. A scalpel was used to shave a portion of the clear coat layer from the vehicle surface, taking care to ensure that no pigment from the underlying basecoat was removed. These samples were analysed without further treatment or preparation.

\section{Infrared Spectroscopy}

Infrared spectra were obtained using a Perkin Elmer ${ }^{\circledR}$ Spectrum 100 FT-IR spectrometer with a Diamond-ZnSe crystal ATR sampling accessory. The ATR accessory was equipped with a pressure arm which was used to maintain the sample and crystal 140 interface contact at a constant force of $80 \mathrm{~N}$. Spectra were acquired over the range of $4000-650 \mathrm{~cm}^{-1}$, with a spectral resolution of $4 \mathrm{~cm}^{-1}$ and eight scans accumulated per sample. Perkin Elmer software (Spectrum ${ }^{\circledR}$ v.6.3.2) was used to perform an ATR correction on the entire dataset to account for the change 145 in the penetration depth with wavelength. Six replicate measurements were obtained at different locations for each sample in order to compare intra-sample vs. inter-sample variations. ${ }^{14}$ The crystal sampling window was thoroughly cleaned using ethanol and lint-free tissue before analysing each 150 sample, and a background scan of the clean crystal obtained between sample acquisitions.

\section{Data Analysis}

Spectra from all samples were subjected to chemometric analysis using the Unscrambler ${ }^{\circledR}$ X software (Camo Software AS, Oslo, 155 Norway). Spectra were truncated to the range of $1800-650 \mathrm{~cm}^{-1}$ as minimal variance in clear coat spectra was observed above $1800 \mathrm{~cm}^{-1}$. The truncated data was baseline corrected and range normalised to remove any variability caused by the sample surface texture, allowing spectra to be visually compared.

160 PCA was performed on the environmental degradation dataset in conjunction with the calibration dataset, and the samples plotted using the first three principal components. This was used to visualise the arrangement and distribution of the samples within the context of the overall model, and to identify any

165 outliers. LDA was then used to predict the classifications for each of the degradation and external test samples. The resulting classifications were compared to expected classifications to evaluate the efficacy of the model. It should be noted that with LDA, all samples will be classified into one of the pre-specified 170 groupings.

\section{Results and Discussion}

\section{Preliminary considerations}

In the previous study by Maric et al., the developed discriminant model was evaluated through test set validation, where the dataset 175 was divided into mutually exclusive training and validation sets. ${ }^{18}$ The training set was used to build the model, which was then used to predict classifications for the validation set. The predicted and actual classifications were then compared to afford a prediction error for the model.

${ }_{180}$ Test set validation is generally considered the most reliable method of validation for chemometric models. ${ }^{27}$ However, division of the dataset reduces the scope of the model when attempting to characterise subsequent samples. For the study outlined in this paper, the original training and validation sets 185 were combined into a single calibration model, utilising the first three principal components and classes derived from PCA of the dataset. The combined model successfully classified $100 \%$ of the calibration data and was used for all subsequent LDA classifications. 


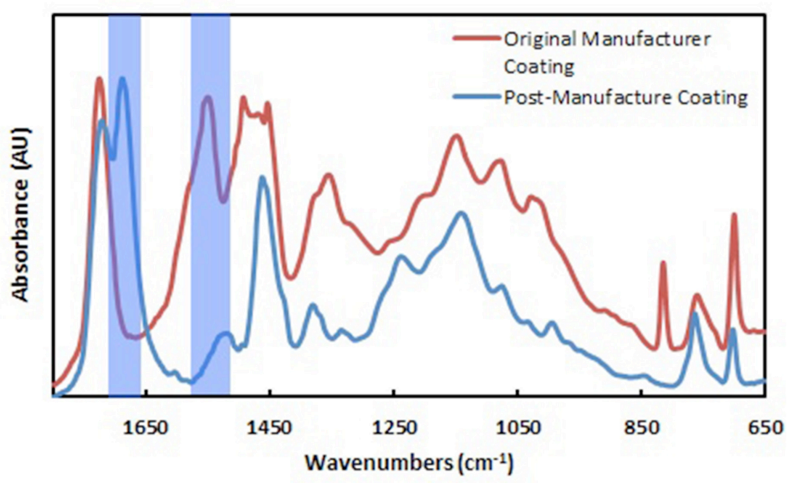

190

Fig. 1 Infrared spectra of automotive paint samples with and without post-manufacture coating. Shaded boxes indicate absorption peaks indicative of polyurethane (ca. $1690 \mathrm{~cm}^{-1}$ ) and melamine (ca. $1550 \mathrm{~cm}^{-1}$ ).

Spectra have been normalised to a maximum absorbance of $1 \mathrm{AU}$.

${ }_{195}$ Expected classifications for each sample were assigned based upon each vehicle's country of manufacture, as determined from the VIN. The VIN is a 17-digit alphanumeric code that provides information regarding the make, model, year and manufacture of a vehicle, which can be decoded using a list of country codes. ${ }^{28,29}$

200 Where the VIN was not available, expected classifications were given based upon the make of the vehicle.

\section{Post-manufacture Clear Coats}

A number of external test samples were found to have a postmanufacture or 'respray' coating applied over the original 205 equipment manufacturer clear coat. These coatings may be applied to conceal degradation or damage to the original paint system, or for aesthetic purposes such as altering the colour or finish of a vehicle. Samples possessing respray coatings were excluded from the model calibration, as they were found to have 210 an unusually high leverage (the distance from the model centre to a projected sample). ${ }^{18}$ This resulted in a bias of the PCA model towards the respray samples, thereby reducing discrimination between the remaining sample classes.

Respray samples are easily recognisable from their infrared 215 spectra, based upon an abnormally high abundance of polyurethane and lack of melamine in comparison to an original manufacturer finish (Figure 1). Polyurethane is a binder characterised by a strong absorption peak near $1690 \mathrm{~cm}^{-1}$, indicative of a carbonyl stretch, ${ }^{30}$ whilst melamine is a cross-

220 linking additive that gives rise to an intense peak at ca. $1550 \mathrm{~cm}^{-1}$ due to an in-plane vibration of its triazine ring. ${ }^{30,31}$ Additionally, respray samples are typically alkyd based rather than acrylic based. Alkyd and acrylic resins are binders which each exhibit absorption bands in the $1300-1000 \mathrm{~cm}^{-1}$ region, ${ }^{30,}{ }^{31}$ however 225 alkyd resins may be distinguished by the absence of clearly defined absorption peaks.

Due to their significantly different chemical composition, these samples were not expected to be accurately described by the existing model calibration. As they are distinguishable by their 230 infrared spectra, these samples may be identified as spectra are obtained (or recognised beforehand using microscopy) and excluded from further analysis. For the purposes of this study, identified respray samples were nonetheless subjected to LDA to investigate whether the model would recognise the samples as 235 atypical.

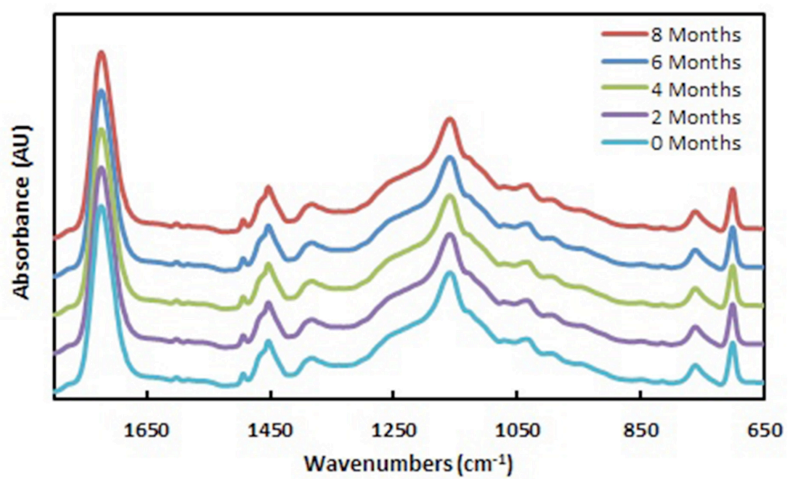

Fig.2 Infrared spectra obtained from an automotive paint sample exposed to Western Australian environmental conditions, following varying months of exposure. Spectra have been normalised to a maximum 240 absorbance of $1 \mathrm{AU}$ and offset for visual clarity.

\section{Characterisation of Degraded Samples}

Climatic conditions in Western Australia vary from tropical or sub-tropical in the northern and southern regions to desert in the central part of the state. ${ }^{32}$ The south-west region in which this ${ }_{245}$ research was conducted is classified as a Mediterranean climate, with dry summers and mild winters. ${ }^{32,33}$

According to meteorological data obtained for the eight-month exposure period, the samples experienced approximately 2200 hours of sunlight, temperatures ranging from $1.6^{\circ} \mathrm{C}$ to $42.5^{\circ} \mathrm{C}$, 250 and $457 \mathrm{~mm}$ of rainfall. ${ }^{34}$

Despite this, visual inspection of the infrared spectra showed no apparent change in clear coat composition over the exposure period, as shown in Figure 2. Absorption peaks characteristic of acrylic binder $\left(1700 \mathrm{~cm}^{-1}\right)$, melamine $\left(1550 \mathrm{~cm}^{-1}\right)$ and styrene $255\left(1490 \mathrm{~cm}^{-1}\right.$ and $\left.1450 \mathrm{~cm}^{-1}\right)$ were consistently observed in each sample, as described in previous work. ${ }^{18}$ It should be emphasised that discrimination between samples is not solely based upon different chemical constituents, but rather on their relative amounts. These differences can be difficult to distinguish through 260 visual comparisons, illustrating the potential of chemometric techniques in discerning between similar samples.

PCA of the exposure samples found no apparent change in the sample groupings over the eight-month period. All spectra were grouped together with the corresponding sample's expected class, 265 as shown in Figure 3.

Although the scores plot revealed some variation in distribution between replicates of each sample, no correlation could be found between the direction of variation and the duration of exposure. A possible explanation is that this variation 270 may have been caused by physical changes in the sample surface texture over the exposure period. Given the small intra-sample variation compared to the inter-sample variation between different classes, it was inferred that the model was still able to clearly distinguish each sample from those of other classes.

275 LDA revealed no change in any of the sample classifications throughout the exposure period, with all spectra correctly assigned to the expected class. Inspection of the discriminant values, which are distance measures of a projected sample from each class's centroid, makes this very clear. Mean discriminant 280 values and their standard deviations for each class are presented in Table 1. Smaller magnitude values signify that samples lie 
closer to a given group's centre.

Throughout the exposure period, the discriminant values for the tested samples showed no variation exceeding the standard ${ }_{285}$ deviation for their respective classes, indicating no change in the distribution of samples. Each sample was found to be within the standard discriminant value range of its assigned class, whilst exhibiting values outside of the standard range for all other classes (see electronic supplementary information Table S1).

290 Table 1 Mean discriminant values and associated standard deviations for each class, derived from calibration dataset.

\begin{tabular}{|c|c|c|c|}
\hline Class Number & $\begin{array}{c}\text { Additional } \\
\text { Manufacture } \\
\text { Details }\end{array}$ & $\begin{array}{c}\text { Mean } \\
\text { Discriminant } \\
\text { Value } \\
\end{array}$ & $\begin{array}{c}\text { Standard } \\
\text { Deviation }\end{array}$ \\
\hline 0 (Australia) & $\begin{array}{c}\text { Manufactured } \\
\text { pre-2009 }\end{array}$ & -6.4 & 3.2 \\
\hline 1 (Japan) & $\begin{array}{l}\text { Toyota, Mazda, } \\
\text { and Mizushima- } \\
\text { manufactured } \\
\text { Mitsubishi } \\
\text { vehicles }\end{array}$ & -5.7 & 2.7 \\
\hline 2 (South Korea) & & -10.1 & 5.3 \\
\hline 3 (Australia) & $\begin{array}{l}\text { Manufactured } \\
\text { post-2009 }\end{array}$ & -3.6 & 1.0 \\
\hline 4 (Japan) & $\begin{array}{l}\text { Subaru, Suzuki, } \\
\text { Honda, and } \\
\text { Nagoya- } \\
\text { manufactured } \\
\text { Mitsubishi } \\
\text { vehicles }\end{array}$ & -9.6 & 5.5 \\
\hline 5 (Mexico) & & -2.4 & 0.1 \\
\hline 6 (Thailand) & & -7.5 & 3.3 \\
\hline 7 (Germany FF) & Ford Focus only & -2.9 & 0.3 \\
\hline 8 (USA) & & -2.4 & 0.2 \\
\hline
\end{tabular}

This implies that each of the samples lies close to their expected group centroid, while being clearly differentiated from the other 295 classes.
These results indicate that environmental exposure over at least an eight-month period does not affect the characterisation of 300 automotive clear coats using the developed chemometric model. This suggests that the model retains potential for classifying samples that have undergone short- to medium-term environmental exposure, and may be used to predict their country of origin and potential manufacturer with a high level of 305 confidence.

\section{External Test Samples}

As a more rigorous test to determine the limitations of the current model, 12 samples originating from heavily weathered vehicles (manufactured pre-2000) were treated as an external test set for 310 model classifications.

Of the 12 samples analysed, one sample originating from a Suzuki Swift was correctly identified as a Class 4 (Japanese) vehicle. However its Class 4 discriminant value was larger than that expected for a Class 4 vehicle based upon the calibration set 315 (supporting information Table S2). This may be due to long-term weathering of the sample, resulting in a subtle change in its characterisation by the model. Nonetheless, the discriminant values show the sample to remain clearly differentiated from the other classes, indicating that this change occurs gradually and 320 may not result in misclassification until a substantial period of exposure has occurred.

11 of the samples were incorrectly classified with regards to the expected classes assigned through each vehicle's make or VIN. Seven of these samples, obtained from six vehicles, were 325 expected to be misclassified due to the presence of a respray coating identified from their infrared spectra. As previously stated, respray samples were excluded from the LDA model calibration in order to retain distinction between other classes. This also results in the model having no information to recognise 330 or define these samples based upon the existing information.
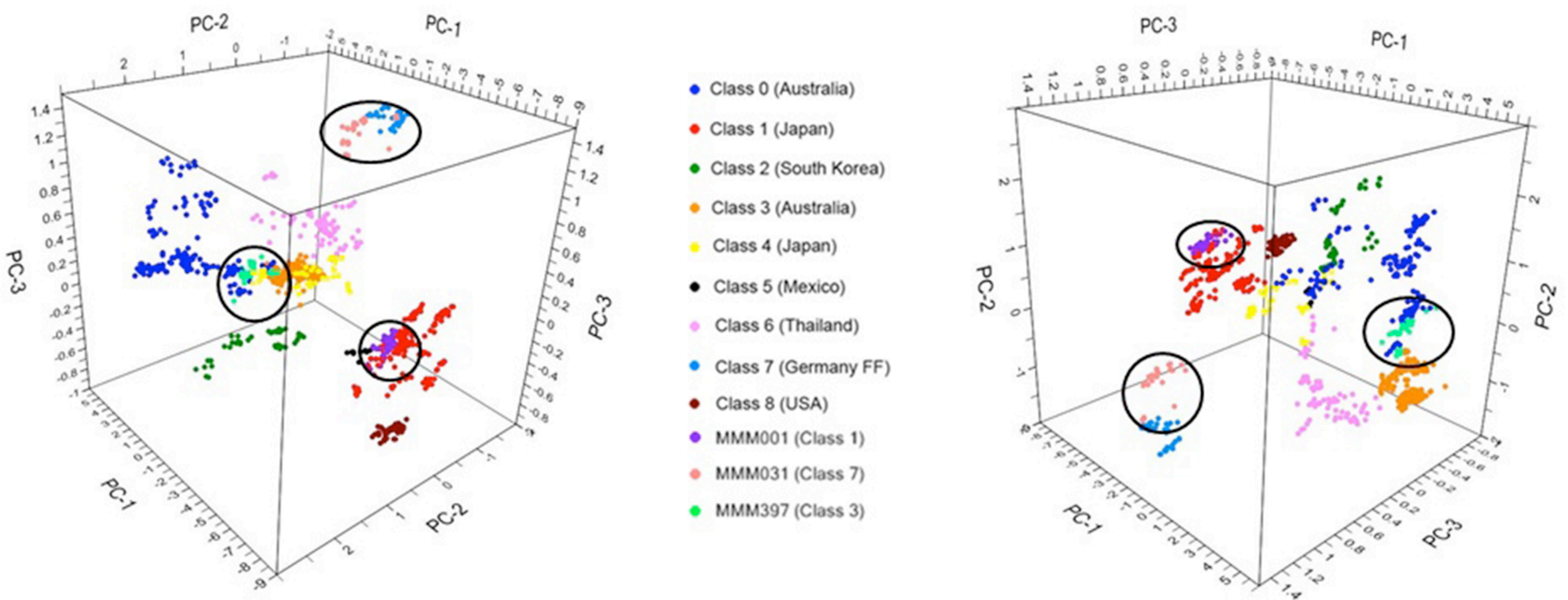

Fig.3 Three-dimensional PCA scores plot showing the distribution of environmental exposure samples (circled) and LDA model calibration samples, based upon their infrared spectra. Class 7 corresponds specifically to the German manufactured Ford Focus. (a) and (b) show different perspectives of the 335 three-dimensional plot. 


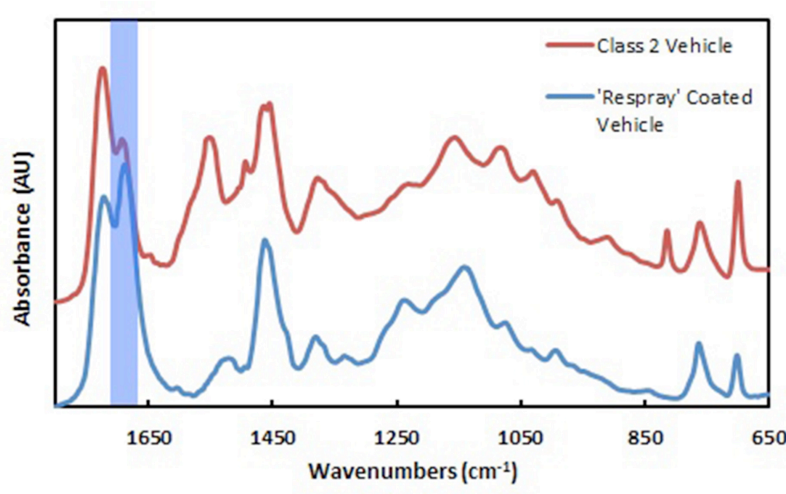

Fig. 4 Infrared spectra from an automotive paint sample with a postmanufacture coating (assigned to Class 2), and a known Class 2 vehicle.

Spectra have been normalised to a maximum absorbance of 1 AU.

Although previously distinguished from their infrared spectra, the respray samples were also identified as atypical through their discriminant values (supporting information Table S3). While each of the samples has been assigned to Class 2, the extremely

345 large magnitude of the discriminant values prove them to be substantially further from the group centroid than a true Class 2 vehicle.

Moreover, the discriminant values obtained against all other classes are sufficiently large to indicate that the samples are 350 clearly beyond the scope of the current model altogether. Interestingly, Class 2 (South Korean) vehicles were the only samples of the calibration dataset in which polyurethane, the main component of respray coatings, was unequivocally detected in the clear coat spectra. ${ }^{18}$ This may explain the classification of

355 these samples with the Class 2 vehicles as a nearest fit, as the samples in both these groups contain polyurethane (Figure 4). It can be seen from the spectra however that the respray samples have a greater relative proportion of polyurethane compared to a true Class 2 vehicle, again making them readily distinguishable.

360 It was noted that the two samples originating from different portions of the Holden Commodore SS vehicle exhibited a considerably greater variation in their discriminant values than observed within the calibration samples. This may originate from different portions of the vehicle experiencing varying levels of 365 exposure to weathering conditions. In this instance the variation did not result in different classifications being assigned. However these results suggest that extremely weathered samples may not give a sufficiently consistent characterisation for model inclusion based upon their clear coat spectra.

370 The remaining four incorrectly classified samples were obtained from a single Daewoo Lanos vehicle originating from South Korea, and did not appear to possess any post-manufacture coating. The incorrect classification of these samples may therefore be due to degradation of the clear coat layer over a 375 significant period of environmental exposure.

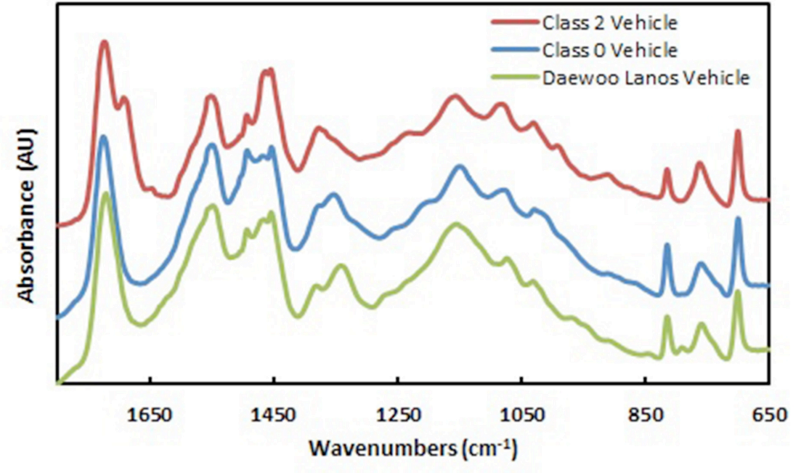

Fig. 5 Infrared spectra obtained from a Daewoo Lanos (expected Class 2) vehicle, a known Class 2 vehicle, and a known Class 0 vehicle. Spectra 380 have been normalised to a maximum absorbance of $1 \mathrm{AU}$.

In particular, despite polyurethane usually being observed in South Korean (Class 2) clear coat samples, no absorption peak was observed near $1690 \mathrm{~cm}^{-1}$ to indicate the presence of polyurethane in the Daewoo Lanos sample spectra (Figure 5). ${ }_{385}$ Polyurethane is known to undergo significant structural changes upon UV exposure, ${ }^{35}$ which may be the cause of the misclassifications for these samples. However, as the composition of these clear coats prior to any environmental exposure is unknown, this cannot be stated with certainty.

390 The discriminant values of the Daewoo Lanos samples suggest them to be fairly close to the Class 0 centroid (supporting information Table S4). Inspection of the corresponding infrared spectra found these samples to be visually indistinguishable from the Class 0 vehicles (Figure 5). As with the Holden Commodore ${ }_{395} \mathrm{SS}$ vehicle, there was also some discrepancy in discriminant values across sections of the vehicle. This may pose an issue when classifying extremely weathered samples, and further work into the effects of long-term degradation on model classifications is required.

\section{${ }_{400}$ Conclusions}

Previous study by Maric et al. developed a chemometric model with a demonstrated ability to predict the likely country of origin for a vehicle based solely on the infrared spectrum of its clear coat layer. While the previous research was conducted using 405 near-factory-finish exemplars, the degradation studies outined in this paper found that environmental exposure over at least an eight-month period does not affect sample classification by the model, which can thus be stated to remain applicable to samples that have undergone short- to medium-term environmental 410 exposure.

Long-term degradation effects however may prevent correct classification and warrant further investigation. In particular, the misclassification of samples that have undergone extreme longterm weathering over at least a 10 -year period is a distinct 415 possibility. The variation in the characterisation of these samples, 
even amongst those taken from the same vehicle, also raise the issue of whether these samples will form a coherent enough group for model inclusion on the basis of their clear coat spectra.

An alternative possibility is that as these samples were taken 420 from extremely old vehicles, there is a high likelihood that the composition of their paint formulations are considerably different to those in current use, resulting in poor classification of these samples by the model. It is therefore possible that incorporating a larger number of vehicles manufactured pre-2000 into the model

425 may better define these samples, and thus provide a greater probability of their correct classification. However inclusion of older vehicles in the model is problematic as the past history of the vehicles would not be known with any certainty.

These results demonstrate that the clear coat cannot always be 430 solely relied upon to give differentiation of automotive paint samples. Sampling should therefore include the procurement of a full cross-section wherever possible, allowing analysis to be made of underlying layers in addition to the clear coat. Further work is currently being undertaken into the use of synchrotron

435 infrared microscopy to analyse chemical components of these underlying layers, and this data may be incorporated into the model to provide more comprehensive classifications.

Samples possessing a post-manufacture or respray coating are a challenge due to their high leverage, which reduces the level of 440 discrimination between original manufacture coatings. However, these samples can be easily identified through visual examination of the infrared spectra as they are acquired, or by microscopic examinations, and excluded from further analysis. Alternatively, these samples can be recognised by inspection of the discriminant

445 values obtained with their LDA classifications. Characterisation of these samples may be possible by targeting the original manufacture coating for analysis, using IR microspectroscopy or other suitable methods.

It should be noted that these studies were conducted using 450 ATR spectroscopy; a rapid and straightforward method of analysis that is already employed routinely in many forensic laboratories. The characterisation methods described in this research can therefore be readily applied within existing analysis schemes. This may be especially valuable in scenarios where 455 automotive paint is the most significant form of physical evidence available to investigators, or where a known sample may not be available for comparison purposes.

Regular expansion of the dataset will be a requirement in order to keep the model up-to-date, as is the case with any forensic 460 database. Automotive paint formulations are constantly being revised, with an increasing shift toward polyurethane-based or solvent-free finishes. ${ }^{36}$ New vehicle makes or models are also constantly being imported into Australia, resulting in a much wider variety of potential samples from international 465 manufacturers. Additionally, there are a number of European countries (such as UK, Sweden or France) that are not yet represented in the model, as the number of vehicles from these manufacturers is currently too small for any generalisations to be made. This will necessitate consistent addition of new data 470 through continued sample collection and/or international collaboration with other institutions.

\section{Acknowledgments}

The authors thank Kelvin Parker and the staff of Prestige 475 Sunroofs (Booragoon, Western Australia); Senior Sergeant Alex Wells and Senior Sergeant Russell Gardiner (Western Australia Police); and the staff at Maddington Auto Wreckers for providing automotive paint samples. Thanks also to Gary Cross (Curtin University) for providing building access for sample exposure 480 studies, and Dr Kari Pitts (ChemCentre) for useful discussions during the course of this research. Georgina Sauzier and Mark Maric are both supported by Australian Postgraduate Awards.

\section{Notes and references}

${ }^{a}$ Department of Chemistry, Curtin University, GPO Box U1987, Perth, 485 Western Australia 6845, Australia,E-mail:S.Lewis@curtin.edu.au; Tel: $+61892662484$

+ Electronic Supplementary Information (ESI) available

1. Strengthening forensic science in the United States: A path forward, Committee on Identifying the Needs of the Forensic Sciences Community, National Research Council, Washington DC, 2009.

2. G. Massonnet and F. Monnard, in Wiley Encyclopedia of Forensic Science, John Wiley \& Sons, Chichester, UK, 2009.

3. J. McCullough, in Wiley Encyclopedia of Forensic Science, John $495 \quad$ Wiley \& Sons, Chichester, UK, 2009.

4. A. R. W. Jackson and J. M. Jackson, Forensic Science, Pearson Prentice Hall, Upper Saddle River, NJ, 2011.

5. R. Saferstein, in Criminalistics: An Introduction to Forensic Science, Pearson Prentice Hall, Upper Saddle River, NJ, 2011.

500 6. J. A. Siegel, Forensic Science: The Basics, Taylor \& Francis, Boca Raton, FL, 2007.

7. E. Bartick, in Handbook of Vibrational Spectroscopy, eds. J. M. Chalmers and P. R. Griffiths, John Wiley \& Sons, New York, 2002.

8. J. Beauchaine, Mikrochimica Acta, 1988, 1, 133-138.

505 9. S. Ryland, T. Jergovich and K. P. Kirkbride, Forensic Science Review, 2006, 18, 97-117.

10. M. Eyring, M. Lovelace and D. Sy, A study of the discrimination of some automotive clear coats having identical color codes, Clearwater Beach, Florida, 2007.

510 11. S. Ryland, Journal of Forensic Sciences, 2001, 46, 31-45.

12. G. Edmondstone, J. Hellman, K. Legate, G. L. Vardy and E. Lindsay, Canadian Society of Forensic Science, 2004, 37, 147-153.

13. E. Liszewski, S. W. Lewis, J. A. Siegel and J. V. Goodpaster, Applied Spectroscopy, 2010, 64, 1122-1125.

515 14. S. L. Morgan and E. G. Bartick, in Forensic Analysis on the Cutting Edge: New Methods for Trace Evidence Analysis, ed. R. D. Blackledge, John Wiley \& Sons, New Jersey, 2007, pp. 333-374.

15. K. Varmuza and P. Filzmoser, Introduction to Multivariate Statistical Analysis in Chemometrics, Taylor \& Francis, Boca Raton, FL, 2009.

520 16. V. Kinton, in Practical Analysis of Flavor and Fragrance Materials, John Wiley \& Sons, 2011, pp. 91-110.

17. J. N. Miller and J. C. Miller, Statistics and Chemometrics for Analytical Chemistry, Pearson Education Limited, London, 2005.

18. M. Maric, W. van Bronswijk, S. W. Lewis and K. Pitts, Analytical 525 Methods, 2012, 4, 2687-2693.

19. J. Bentley, in Forensic Examination of Glass and Paint, CRC Press, New York, 2001. 
20. C. A. Smith, J. L. Gerlock and R. O. Carter, Polymer Degradation and Stability, 2001, 72, 89-97.

530 21. D. R. Bauer, Polymer Degradation and Stability, 2000, 69, 297-306.

22. J. F. Larché, P. O. Bussière and J. L. Gardette, Polymer Degradation and Stability, 2010, 95, 1810-1817.

23. U. Schulz, P. Trubiroha, U. Schernau and H. Baumgart, Progress in Organic Coatings, 2000, 40, 151-165.

535 24. M. Mohseni, B. Ramezanzadeh and H. Yari, in New Trends and Developments in Automotive Industry, ed. M. Chiaberge, InTech, 2011.

25. W.-T. Chang, C.-C. Yu, C.-T. Wang and Y.-Y. Tsai, Forensic Science Journal, 2003, 2, 47-58.

540 26. W. Chang, T. Chen, C. Yu and J. Kau, Forensic Science Journal, $2002,55-60$.

27. R. G. Brereton, in Applied Chemometrics for Scientists, John Wiley \& Sons, 2007, pp. 145-191.

28. AIAM, Passenger Vehicle Identification Handbook, 2000.

${ }_{545} 29$. W. T. Smylie, in Forensic Investigation of Stolen-Recovered and Other Crime-Related Vehicles, eds. E. Stauffer and M. S. Bonfanti, Academic Press, Oxford, 2006.

30. A. Beveridge, T. Fung and D. MacDougall, in Forensic Examination of Glass and Paint, CRC Press, New York, 2001.

550 31. P. Buzzini and W. Stoecklein, in Encyclopedia of Analytical Science, eds. P. Worsfold, A. Townshend and C. Poole, Elsevier, Oxford, 2nd edn., 2005, pp. 453-464.

32. Bureau of Meteorology, Australian climate zones - Major classification groups,

555 http://www.bom.gov.au/climate/environ/other/kpn_all.shtml, Accessed 10 March, 2013.

33. Bureau of Meteorology, Climate education: Climate of Western Australia,

http://www.bom.gov.au/lam/climate/levelthree/ausclim/ausclimwa.ht

560 m, Accessed 13 March, 2013.

34. Bureau of Meteorology, Climate Data Online, http://www.bom.gov.au/climate/data/, Accessed 18 September, 2012.

35. D. Rosu, L. Rosu and C. N. Cascaval, Polymer Degradation and Stability, 2009, 94, 591-596.

565 36. N. N. Daeid and M. M. Houck, Interpol's Forensic Science Review, Taylor \& Francis, Boca Raton, FL, 2010. 